\title{
Azimuthal velocity shear within an Earthward fast flow - further evidence for magnetotail untwisting?
}

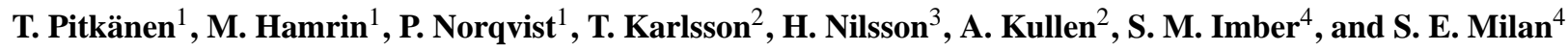 \\ ${ }^{1}$ Department of Physics, Umeå University, Umeå, Sweden \\ ${ }^{2}$ Space and Plasma Physics, School of Electrical Engineering, Royal Institute of Technology, Stockholm, Sweden \\ ${ }^{3}$ Swedish Institute of Space Physics, Kiruna, Sweden \\ ${ }^{4}$ Department of Physics and Astronomy, University of Leicester, Leicester, UK \\ Correspondence to: T. Pitkänen (timo.pitkanen@space.umu.se)
}

Received: 12 November 2014 - Revised: 2 February 2015 - Accepted: 3 February 2015 - Published: 3 March 2015

\begin{abstract}
It is well known that nonzero interplanetary magnetic field $B_{y}$ conditions lead to a twisted magnetotail configuration. The plasma sheet is rotated around its axis and tail magnetic field lines are twisted, which causes an azimuthal displacement of their ionospheric footprints. According to the untwisting hypothesis, the untwisting of twisted field lines is suggested to influence the azimuthal direction of convective fast flows in the nightside geospace. However, there is a lack of in situ magnetospheric observations, which show actual signatures of the possible untwisting process. In this paper, we report detailed Cluster observations of an azimuthal flow shear across the neutral sheet associated with an Earthward fast flow on 5 September 2001. The observations show a flow shear velocity pattern with a $V_{\perp y}$ sign change, near the neutral sheet $\left(B_{x} \sim 0\right)$ within a fast flow during the neutral sheet flapping motion over the spacecraft. Firstly, this implies that convective fast flows may not generally be unidirectional across the neutral sheet, but may have a more complex structure. Secondly, in this event tail $B_{y}$ and the flow shear are as expected by the untwisting hypothesis. The analysis of the flow shear reveals a linear dependence between $B_{x}$ and $V_{\perp y}$ close to the neutral sheet and suggests that Cluster crossed the neutral sheet in the dawnward part of the fast flow channel. The magnetospheric observations are supported by the semi-empirical T96 and TF04 models. Furthermore, the ionospheric SuperDARN convection maps support the satellite observations proposing that the azimuthal component of the magnetospheric flows is enforced by a magnetic field untwisting. In summary, the observations give strong supportive evidence to the tail untwisting hypothesis. However, the
\end{abstract}

T96 ionospheric mapping demonstrates the limitations of the model in mapping from a twisted tail.

Keywords. Magnetospheric physics (magnetospheric configuration and dynamics; magnetotail; plasma convection)

\section{Introduction}

Theoretical considerations (Russell, 1972; Cowley, 1981), observations (e.g. Kaymaz et al., 1994; Owen et al., 1995; Tsyganenko et al., 1998; Tsyganenko and Fairfield, 2004) as well as simulations (e.g. Kaymaz et al., 1995; Kullen and Janhunen, 2004a, b; Walker et al., 1999) show that nonzero interplanetary magnetic field (IMF) $B_{y}$ conditions lead to a twisted magnetotail configuration. The entire tail plasma sheet is rotated around its axis and tail magnetic field lines are twisted from the north-south symmetry: an additional $B_{y}$ term collinear to the IMF $B_{y}$ appears in the tail (Fairfield, 1979), with higher values in the plasma sheet than the lobes and the maximum close to the neutral sheet (e.g. Kaymaz et al., 1994; Kullen and Janhunen, 2004a, b; Lui, 1986).

The twisting is expected to be closely associated with plasma convection in the nightside geospace. Observations of nightside ionospheric convection have shown that fast transient azimuthal flow bursts are regularly seen in the midnight sector specifically during northward and $B_{y}$-dominating IMF and nonsubstorm conditions (e.g. Grocott et al., 2003, 2004). These flow bursts have been suggested to be a manifestation of the untwisting process of the twisted tail field lines during the magnetic field reconfiguration following reconnection in a twisted tail (Grocott et al., 2004). 


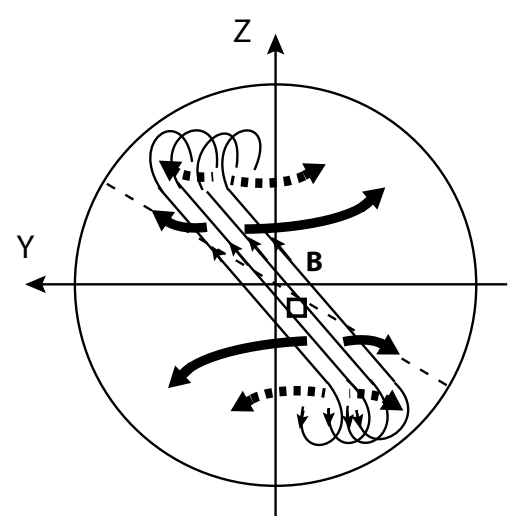

Figure 1. Schematic illustration of the untwisting of tail field lines under a positive IMF $B_{y}$ exposure (positive twisting), when viewed from the tail towards the Earth. The thick solid and dashed arrows indicate the expected azimuthal convection directions in the magnetotail and the conjugate convection flows in the ionosphere, respectively. The thin dashed line marks the tilted neutral sheet. The black square marks a satellite position at an azimuthal flow reversal within the Earthward transported untwisting field lines (see Fig. 2) (after Grocott et al., 2005).

According to the tail untwisting hypothesis by Grocott et al. (2004), tail twisting introduced by a prolonged exposure to significant IMF $B_{y}$ causes the ionospheric footprints of a reconnecting tail field line to be azimuthally displaced in the Northern and Southern Hemispheres. Consequently, the newly reconnected field line will have to travel faster in one hemisphere to convect, via dusk or dawn, back towards dayside. This gives rise to the fast azimuthal flow bursts observed in the nightside ionosphere. The situation of the untwisting of magnetic field lines in a positively twisted tail (the field lines twisted counter-clockwise and the neutral sheet rotated clockwise around the Sun-Earth line when viewed from downtail looking Earthward) according to the hypothesis is illustrated in Fig. 1.

Interhemispheric observations of ionospheric azimuthal flow bursts support the above idea of untwisting and have confirmed that the asymmetric convection patterns in the Northern and Southern Hemispheres are resembling mirror images of each other (Grocott et al., 2005). In addition, Grocott et al. (2007) have presented simultaneous in situ magnetospheric observations made when these flow bursts were seen in the ionosphere. The observations showed that these ionospheric azimuthal flow bursts were associated with Earthward fast flows, which had a convective azimuthal velocity component matching the ionospheric measurements.

Recently, motivated by the results by Grocott et al. (2007), Pitkänen et al. (2013) using Cluster data conducted a statistical investigation of a possible IMF dependence (and hence twist-dependence) of the direction of the azimuthal convection velocity component of the Earthward magnetotail fast flows. It was found that the azimuthal flow direction of the fast flows and the orientation of the IMF show a correlation and in such a manner that is consistent with the untwisting hypothesis. Surprisingly, the correlation was found to be quite strong regardless of the IMF $B_{z}$ direction. One could expect stronger correlation for northward IMF conditions, since ionospheric azimuthal flow bursts are observed mainly during northward and $B_{y}$ dominating IMF. For southward IMF, clearly azimuthally oriented ionospheric flow bursts in the midnight sector are much less frequent (Grocott et al., 2008). Also, it has been shown both observationally (Owen et al., 1995; Tsyganenko and Fairfield, 2004) and in simulations (e.g. Walker et al., 1999; Kullen and Janhunen, 2004a, b) that the twist of the tail plasma sheet around its axis is considerably stronger for northward than for southward IMF. Whether this is true even for the degree of magnetic field line twisting remains to be shown. One possible interpretation of the results by Pitkänen et al. (2013) is that the untwisting process could be occurring in the tail to some extent for all nonzero IMF $B_{y}$ conditions, and not only limited to those of northward and $B_{y}$ dominating IMF. This possibility should be studied further.

The untwisting hypothesis predicts a generation of an azimuthal flow shear by the Earthward transported untwisting magnetic field lines. Figure 2 illustrates the situation for positive tail twisting. In the figure the black square indicates a spacecraft that would observe an azimuthal velocity reversal when moving in the north-south direction across the neutral sheet. According to the hypothesis, the flow reversal is expected to be located at or close to the neutral sheet for untwisting field lines near local midnight. Farther away from midnight, i.e. towards the dawn and dusk flanks of the region of untwisting field lines, the flow reversal is predicted to be situated above or below the neutral sheet: the division between dawnward and duskward flows is the interface between the dawnward and duskward moving field lines, which is inclined relative to the tilted neutral sheet. Figure 1 shows the situation for positive tail twisting, in the case where the flow reversal is located below and above the neutral sheet dawnward and duskward of the centremost untwisting field lines, respectively (see also Grocott et al., 2007, their Fig. 6c). Note that in such a situation it is expected that the tail field lines are twisted significantly out of a meridian plane and have a fairly small inclination angle to the neutral sheet.

However, there is a lack of reported observations of the flow shear and the untwisting process in general in the magnetosphere. Some indications of a flow shear have been suggested to be observed by Grocott et al. (2007). Their observations from either side of the tail neutral sheet showed convective flows with opposite azimuthal sense in the opposite sides of the neutral sheet, which suggests closed field lines twisted significantly out of a meridian plane. In addition to that case, which occurred during northward and $B_{y}$-dominating IMF conditions, Walsh et al. (2009) have reported an azimuthal flow shear across the neutral sheet in association with an 


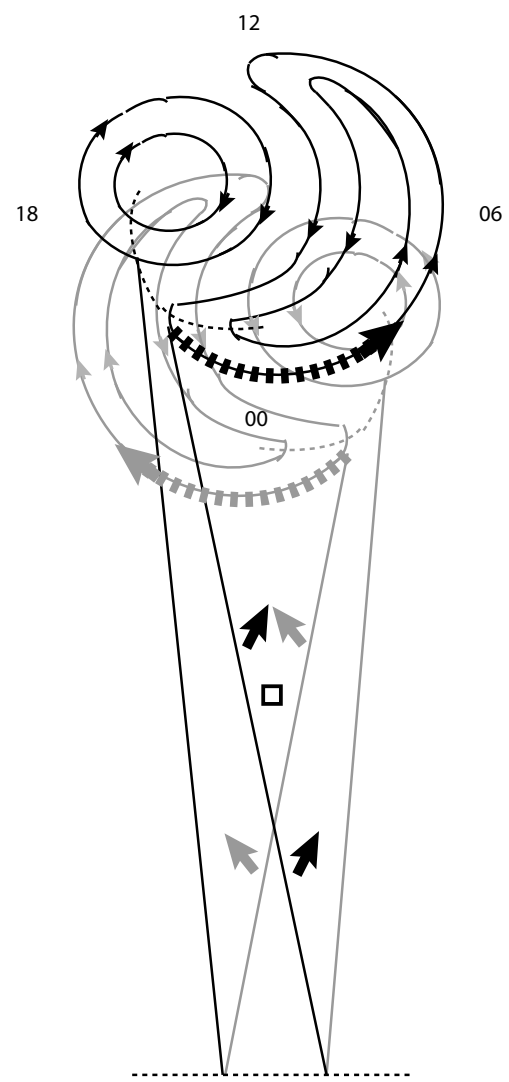

Figure 2. Schematic illustration of the untwisting of positively twisted tail field lines when looking down from the north. The dotted lines indicate the reconnection line in the nightside ionosphere and in the magnetotail. The solid arrowed curves show the ionospheric convection streamlines and the thick dashed arrows depict the azimuthal flow bursts associated with the untwisting. The straight lines represent field lines, which connect the two hemispheres via the reconnection line. The short solid arrows indicate the directions of convective magnetotail fast flows associated with the untwisting. For clarity, the Northern and Southern Hemispheres are coloured by black and grey, respectively, and the ionospheric convection patterns are slightly separated. The black square marks a satellite position in the region where an azimuthal flow shear with a reversal generated by the untwisting field lines can be observed if the satellite is moving in the north-south direction across the neutral sheet (after Grocott et al., 2005).

Earthward fast flow. This event occurred during southward and $B_{y}$-dominating IMF conditions. Walsh et al. (2009) suggested that the flow shear could be related to untwisting, but could not rule out a possibility of it to be related to internal flow patterns within the fast flow channel (depleted bubble), since the flow shear was observed only by one spacecraft. Besides these two studies, also Pitkänen et al. (2013) have reported an indication of an azimuthal velocity shear across the neutral sheet during a fast flow in their fast flow example event. However, they showed only single satellite measurements and did not discuss the event any further. It is clear a)
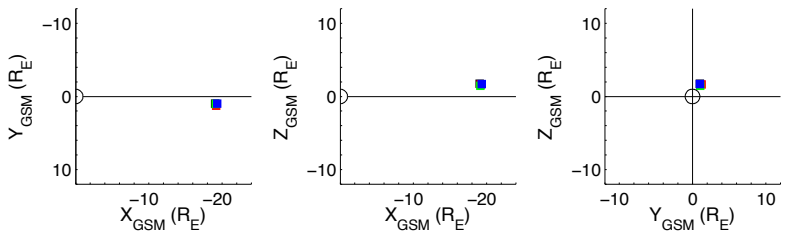

b)
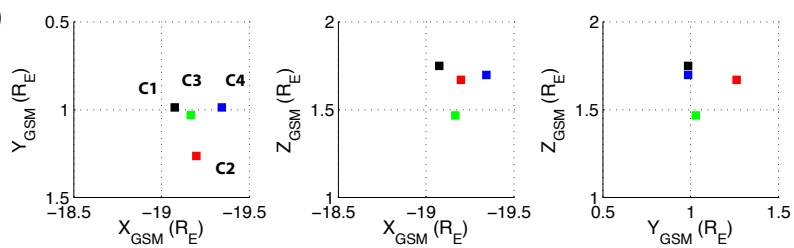

Figure 3. (a) Cluster spacecraft positions in GSM on 5 September 2001 at 11:26 UT. (b) Zoomed view.

that detailed observations about these fast flow-associated azimuthal flow shears with reversals are needed to understand their nature and how they are related to a magnetotail fast flow and possible untwisting of closed field lines in the magnetotail.

In this paper, we analyse and discuss the flow shear event identified by Pitkänen et al. (2013) in more detail. We will extend their preliminary single spacecraft analysis by taking the full advantage of the Cluster multispacecraft observations. In addition, ionospheric convection measurements by the $\mathrm{Su}-$ perDARN network are used for analysing the Cluster observations in terms of the untwisting hypothesis. The paper is organized as follows: the instrumentation used is described in Sect. 2, the flow shear event is presented in Sect. 3 and discussed in Sect. 4, and the summary of the results together with conclusions are given in Sect. 5.

\section{Instrumentation}

The magnetospheric observations for this study are made by the Cluster satellites (C1-C4). We use spin-resolution $(\sim 4 \mathrm{~s})$ velocity data from the Hot Ion Analyser (HIA) detector $(\mathrm{C} 1$ and $\mathrm{C} 3)$ and from the COmposition DIstribution Function (CODIF) detector (C4) of the Cluster Ion Spectrometry (CIS) instrument (Rème et al., 2001). In addition, 3 -spin resolution $(\sim 12 \mathrm{~s})$ omni-directional ion energy distribution data in particle energy flux units from the C1 CIS HIA detector are presented. Magnetic field data, as well as spin resolution, come from the FluxGate Magnetometer experiment (FGM) (Balogh et al., 2001). Since the C2 spacecraft has no operational CIS instrument onboard, we do not show any data from the $\mathrm{C} 2$ satellite. IMF data presented in this study are $1 \mathrm{~min}$ ACE satellite measurements propagated to the bow shock nose provided by the OMNI database (http://omniweb.gsfc.nasa.gov/). The IMF and magnetospheric data are presented in the geocentric solar magnetic (GSM) coordinates throughout the study if not stated otherwise. 


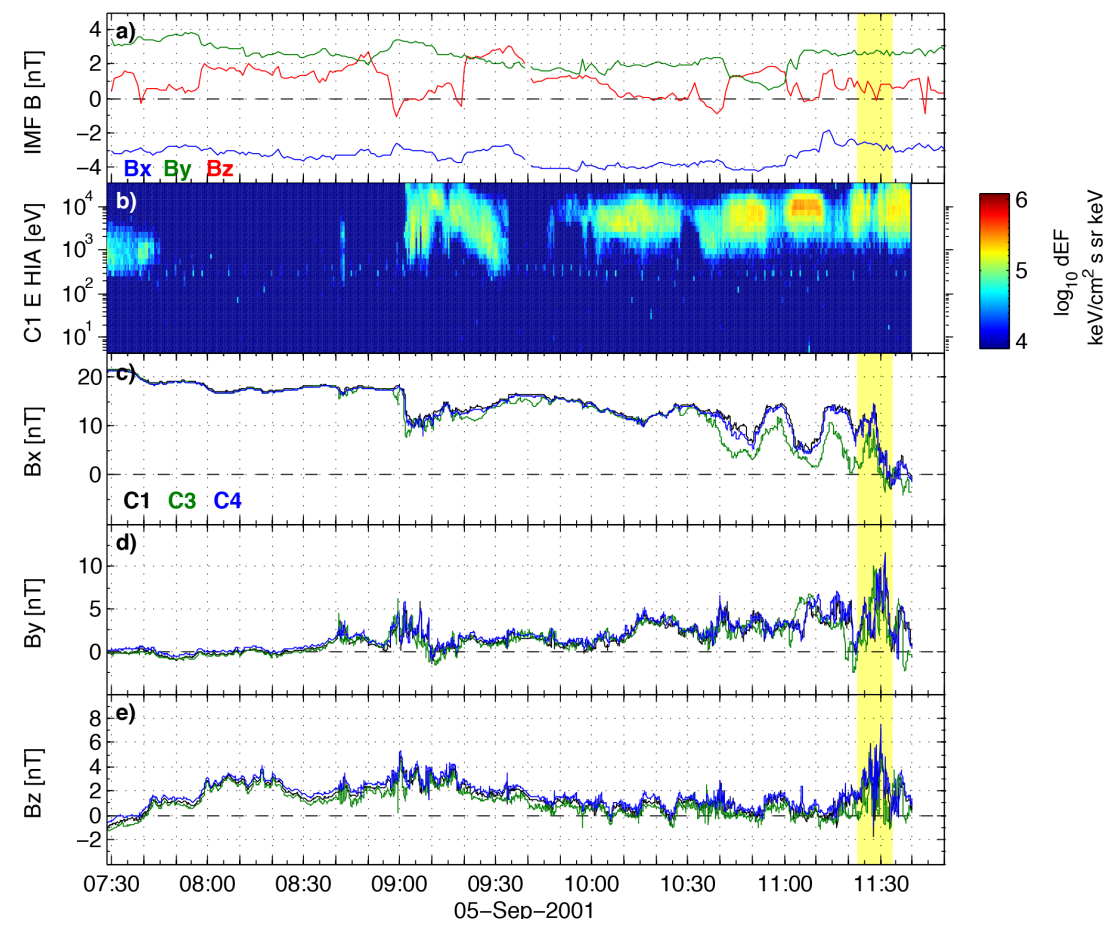

Figure 4. (a) IMF components, (b) Cluster C1 HIA omni-directional ion energy spectrogram, and (c-e) Cluster $B$ field components during the $\sim 4 \mathrm{~h}$ period prior the fast flow event occurring at $\sim 11: 22-11: 34$ UT on 5 September 2001 . The time interval of the fast flow event is marked by yellow shading.

The ionospheric observations used in this study are $2 \mathrm{~min}$ large-scale ionospheric convection maps provided by SuperDARN (Greenwald et al., 1995; Chisham et al., 2007). SuperDARN is an international network of HF radars located around the polar regions of both hemispheres. The radars provide continuous monitoring of the large-scale horizontal ionospheric plasma flow in the polar ionospheres and their data are hence suitable for comparison with flow observations from Cluster. The SuperDARN convection maps are derived by fitting a solution for the electrostatic potential, which is expressed in spherical harmonics, to the line-ofsight velocity measurements ("Map Potential" model; Ruohoniemi and Baker, 1998). The equipotentials of the solution represent the plasma streamlines of the modelled convection pattern. Where no measurement points are available, information from the statistical model of Ruohoniemi and Greenwald (1996), parameterized by concurrent IMF conditions, is used.

\section{Observations}

\subsection{Overview}

The fast flow event occurred on 5 September 2001 at about 11:22-11:34 UT. According to the AE indices, the event was associated with no substorm activity (data not shown). Figure 3 displays the Cluster satellite positions at 11:26 UT. The spacecraft were located in the premidnight sector close to the midnight meridian at a distance of $\sim-19 R_{\mathrm{E}}$ in XGSM (C3 position at 11:26 UT: $[-19.2,1.0,1.5] R_{\mathrm{E}}$ ).

Figure 4 shows the IMF components, Cluster $\mathrm{C} 1$ ion spectrogram, and the $B$ field components measured by Cluster $\mathrm{C} 1, \mathrm{C} 3$ and $\mathrm{C} 4$ during the $4 \mathrm{~h}$ prior to the fast flow event. From Fig. 4a we note that the IMF was quite stable during that period. The IMF $B_{x}$ was negative and the IMF $B_{y}$ positive during the entire period. The IMF $B_{z}$ was mainly positive, i.e. northward, but had occasional short excursions southward. Also, except for a couple of short intervals, the IMF $B_{y}$ magnitude was greater than the IMF $B_{z}$ magnitude. The IMF conditions during the period could be hence characterized as northward and $B_{y}$-dominating conditions.

From the Cluster magnetic field data we note that the satellites were mainly traversing field lines in the Northern Hemisphere (Fig. 4c). The ion spectrogram from C1 (Fig. 4b) indicates that after staying in the lobe practically continuously for $\sim 75 \mathrm{~min}$, Cluster entered and stayed on closed field lines after 09:00 UT (except 09:34:29-09:45:57 UT) beyond the fast flow event at least until $\sim 11: 40 \mathrm{UT}$, after which the Cluster data become unavailable. Figure $4 \mathrm{~d}$ shows that the $B_{y}$ at all three spacecraft turned positive after 08:30 UT and stayed practically positive for more than $3 \mathrm{~h}$ including the fast flow event. The Cluster $B_{y}$ was hence collinear with the IMF $B_{y}$ for several hours prior to the fast flow event both on open and closed field lines, which is in agreement with observa- 
tions of a $B_{y}$ component in the tail due to the influence of nonzero IMF $B_{y}$ (e.g. Fairfield, 1979; Kaymaz et al., 1994) and suggests a twisting of closed field lines. In a nontwisting situation, the tail magnetic field in the premidnight Northern Hemisphere would be expected to have a negative $B_{y}$ due to the azimuthal tail field line flaring. The $B_{y}$ and $B_{z}$ components also generally show gradual increasing and decreasing trends, respectively, during this period. These could indicate a loading of the magnetotail analogous to a substorm growth phase being associated with the twisting. The observed fast flow would then be a natural manifestation of a localized tail relaxation process. The enhancements of the $B_{y}$ and $B_{z}$ during the event are related to the magnetic perturbations associated with the fast flow.

When looking at the Cluster $B_{x}$ components in more detail in Fig. 4c, we notice that after 10:30 UT the $B_{x}$ at all spacecraft show oscillations. The oscillations at $\mathrm{C} 3$ reach closer to the neutral sheet than those of $\mathrm{C} 1$ and $\mathrm{C} 4$ due to $\mathrm{C} 3$ being the southernmost of the spacecraft (middle panel in Fig. 3b). These oscillations have periods of $\sim 8$ to $\sim 27 \mathrm{~min}$. We interpret that these $B_{x}$ oscillations could be related to neutral/current sheet flapping (e.g. Zhang et al., 2005, 2002; Sergeev et al., 2004).

Figure 5 shows the Cluster location in GSM at 11:26 UT together with the magnetic field line of the T96 model (Tsyganenko, 1995) traced through the Cluster position. For clarity, only the $\mathrm{C} 3$ position is shown. From Fig. $5 \mathrm{~b}$ we note that the T96 field line is bent or twisted from the meridian direction in such a way that it has a net positive $B_{y}$ component at large geocentric distances and in the region where Cluster is located. The twist of the $B$ field line is not uniform, but it is stronger close to the neutral sheet. These imply that the twisting of the tail field lines is also produced in the T96 model, and it gives support to our Cluster interpretation that there exists a twist in the tail during the event. However, note that the T96 model suggests that C3 at 11:26 UT would be located at the apex of the field line close to the neutral sheet (Fig. 5a). This actually is not the case as can be seen from the magnetic field data presented in Fig. 6, that will be discussed in the next section and which shows that $\mathrm{C} 3$ (and other Cluster spacecraft as well) are clearly above the neutral sheet at that time.

The thick black line in Fig. 5b indicates the neutral sheet according to the TF04 neutral sheet model (Tsyganenko and Fairfield, 2004). Note that the model neutral sheet here is presented using the geocentric solar wind (GSW) coordinate system, which differs from the GSM system in such a way that its $X$ axis is antiparallel to the direction of the solar wind. The neutral sheet is plotted at the C3 XGSW distance where the C3 XGSW coordinate is approximated by its XGSM coordinate. From Fig. $5 \mathrm{~b}$ we notice that the model neutral sheet is weakly rotated clockwise around the $X$-axis: The normal of the neutral sheet creates an angle of $\sim 2$ degree with the $Z$-axis in the $Y Z$ plane (not marked in the figure). The clockwise rotation, even though weak, is consistent with the ex-
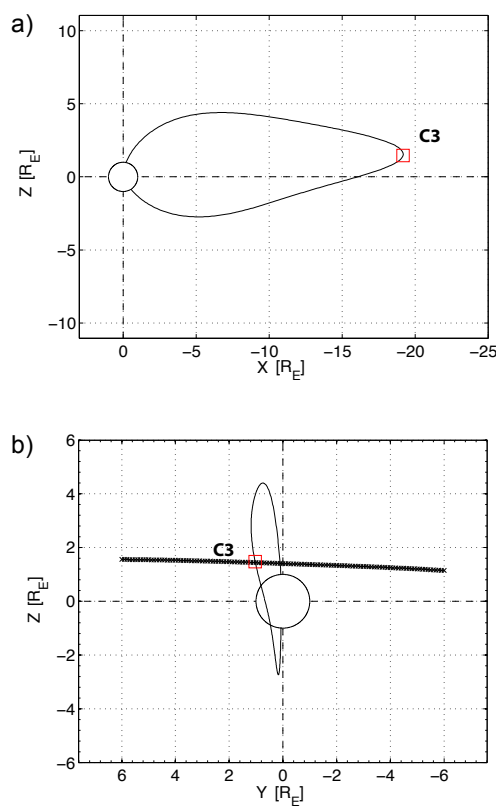

Figure 5. (a-b) Location of the Cluster 3 spacecraft (red square) in the GSM coordinate system at 11:26 UT on 5 September 2001. The solid loop indicates the T96 model field line traced through the spacecraft position. (b) The thick black line indicates the TF04 model neutral sheet in the GSW coordinate system at the C3 XGSW distance where the $\mathrm{C} 3 \mathrm{XGSW}$ coordinate is approximated by its XGSM coordinate.

pected neutral sheet rotation direction for positive tail twisting (e.g. Cowley, 1981) and gives additional support for the tail twisting during the event.

\subsection{The flow event: Cluster}

In Fig. 6 we present the Cluster magnetic field data and velocity data from the time interval of the fast flow event. The panels in the figure from top to bottom are the following: (a) $X$ components $\left(B_{x}\right)$ of the magnetic field for $\mathrm{C} 1$ (black), $\mathrm{C} 3$ (green) and $\mathrm{C} 4$ (blue); (b) $Y$ components $\left(B_{y}\right)$ of the magnetic field for $\mathrm{C} 1, \mathrm{C} 3$ and $\mathrm{C} 4$; (c) $Z$ components $\left(B_{z}\right)$ of the magnetic field for $\mathrm{C} 1, \mathrm{C} 3$ and $\mathrm{C} 4$; (d) $X$ and $Y$ components perpendicular to the magnetic field ( $V_{\perp x}$ and $V_{\perp y}$, solid lines) of the ion bulk velocity and the $X$ component of the ion bulk velocity ( $V_{x}$, dashed line) for $\mathrm{C} 1$; (e) same as in (d) for $\mathrm{C} 3$; and (f) $X$ and $Y$ components perpendicular to the magnetic field of the proton bulk velocity (solid lines) and the $X$ component of the proton bulk velocity (dashed line) for $\mathrm{C} 4$.

The Earthward fast flow event begins at $\sim 11: 23 \mathrm{UT}$. The event is associated with three major flow bursts $\left(>200 \mathrm{~km} \mathrm{~s}^{-1}\right)$ seen in the $V_{x}$ at $\mathrm{C} 1$ and $\mathrm{C} 4$ and a similar longer continuous one at $\mathrm{C} 3$ during the next $10 \mathrm{~min}$ (blue dashed lines in Fig. 6d-f). At first the flow in the $X$ direction at all satellites has a significant field-aligned component, but later it turns to pure perpendicular, as can be seen from the 


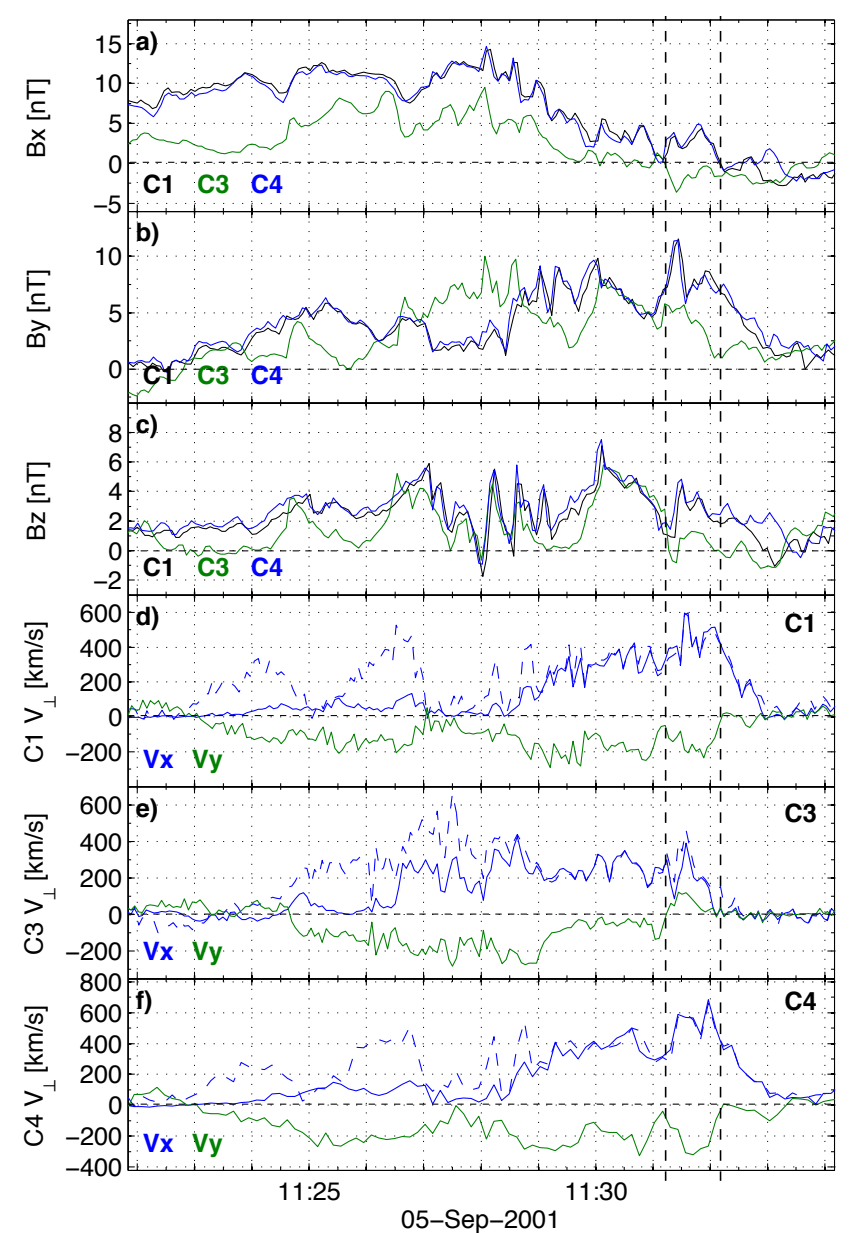

Figure 6. Cluster data on 5 September 2001 at 11:22-11:34 UT. (ac) Magnetic field components measured by the $\mathrm{C} 1$ (black line), $\mathrm{C} 3$ (green line) and $\mathrm{C} 4$ (blue line) spacecraft. (d-e) $X$ and $Y$ components of the ion bulk velocity for $\mathrm{C} 1$ and $\mathrm{C} 3$ from the HIA detector. (f) $X$ and $Y$ components of the proton bulk velocity for $\mathrm{C} 4$ from the CODIF detector. The dashed and solid lines mark the components of the total velocity and the velocity perpendicular to the magnetic field, respectively. The first vertical dashed line indicates the $V_{\perp y}$ reversal associated with the $B_{x}$ sign change at $\mathrm{C} 3$. The second vertical dashed line indicates the $V_{\perp y}$ reversal associated with the first $B_{x}$ sign change at $\mathrm{C} 1$ and $\mathrm{C} 4$.

difference between the $V_{x}$ and $V_{\perp x}$ curves (blue dashed and solid lines, respectively). This is consistent with the fact that the satellites resided first farther out from, and then at a later stage moved closer to, the neutral sheet (Fig. 6a). It is known that the fast flows have a substantial convective component in and a large field-aligned component further away from the central plasma sheet (e.g. Petrukovich et al., 2001; Raj et al., 2002).

From Fig. $6 \mathrm{~d}-\mathrm{f}$ we notice that the $V_{\perp y}$ is mostly negative during the event at all spacecraft, i.e. the flow has a dawnward component. However, at 11:31:13 UT the C3 velocity data show an interesting feature (Fig. 6e, first vertical dashed

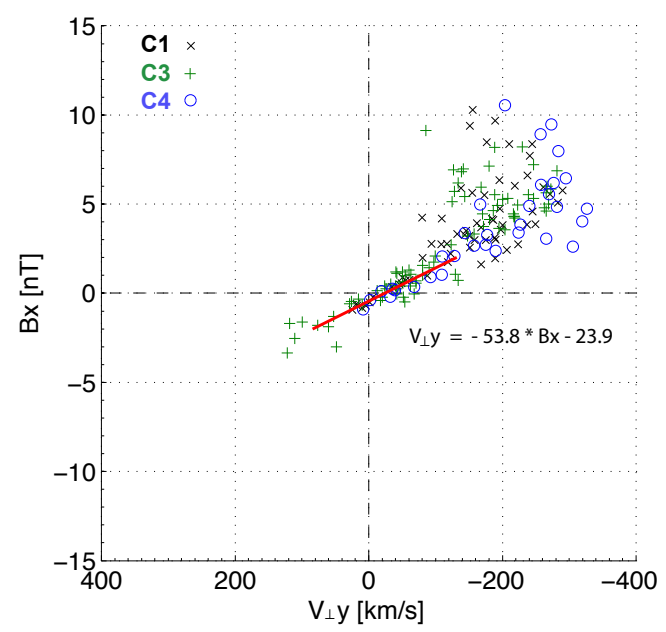

Figure 7. $V_{\perp y}$ versus $B_{x}$ during the main Earthward flow (continuously $V_{\perp x}>100 \mathrm{~km} \mathrm{~s}^{-1}$ ) for each spacecraft. The red line indicates a linear fit to data points close to the neutral sheet $\left(-2 \leq B_{x} \leq 2 \mathrm{nT}\right)$.

line). The $V_{\perp y}$ component turns from negative to positive, i.e. from dawnward to duskward. From Fig. 6a, we notice that the $V_{\perp y}$ sign change is associated with the $B_{x}$ change from positive to negative, i.e. the neutral sheet was flapping over the spacecraft. At 11:32:10 UT, C1 and C4 cross the neutral sheet (second vertical dashed line) at higher ZGSM coordinates and also these spacecraft move onto southern field lines for a short moment. Again, the $B_{x}$ sign change from positive to negative is associated with the direction change of the azimuthal velocity component $V_{\perp y}$ from negative to positive, i.e. from dawnward to duskward direction, even though the magnitudes of the duskward $V_{\perp y}$ components for $\mathrm{C} 1$ and especially for $\mathrm{C} 4$ here are small.

In Fig. 7 we present the flow shear observed by each of the three spacecraft as a scatter plot of $V_{\perp y}$ versus $B_{x}$. For each spacecraft, we have plotted the data points over the time interval of the main convective Earthward flow (continuously $V_{\perp x}>100 \mathrm{~km} \mathrm{~s}^{-1}$ excluding one and two points below this threshold for $\mathrm{C} 1$ and $\mathrm{C} 3$, respectively). The scatter plot shows a clear pattern: above the neutral sheet the flow has a dawnward component, and below a duskward component.

Thus, the multisatellite Cluster data presented in Figs. 6 and 7 show that an azimuthal flow shear with a $V_{\perp y}$ sign change may take place in the vicinity of the neutral sheet $\left(B_{x} \sim 0\right)$ within a fast flow. Since we are dealing with bulk flow perpendicular to the magnetic field, this implies that (assuming the frozen-in condition) magnetic field lines are in overall convecting to opposite azimuthal directions above and below the neutral sheet within the studied fast flow.

If we view the azimuthal flow directions in the framework of the tail untwisting hypothesis, we notice that the directions of the azimuthal flows are in agreement with the hypothesis. For positive IMF $B_{y}$ conditions and hence for positive twist- 
ing of the tail field lines, in the midnight sector observed fast flow is expected to have predominantly a dawnward component in the Northern Hemispheric and a duskward component in the Southern Hemispheric field lines. The situation is schematically illustrated in Fig. 2.

However, the scatter plot reveals an interesting feature that is not directly obvious from Fig. 6 . The change of the $V_{\perp y}$ sign does not occur precisely at but slightly below the neutral sheet. The $B_{x}$ value at the $V_{\perp y}$ sign change is $\sim-0.4 \mathrm{nT}$. There is also an indication of higher velocities further out from the neutral sheet with a linear dependence close to the neutral sheet. The red line in Fig. 7 indicates a linear fit to data points from all three spacecraft close to the neutral sheet $\left(-2 \leq B_{x} \leq 2 \mathrm{nT}\right)$. The fit gives a relation $V_{\perp y}=-53.8 \cdot B_{x}-23.9$, where $V_{\perp y}$ and $B_{x}$ are given in $\mathrm{km} \mathrm{s}^{-1}$ and $\mathrm{nT}$, respectively.

A growing offset between the $V_{\perp y}$ reversal and the neutral sheet farther away from midnight, i.e. towards the dawn and dusk flanks of the region of untwisting field lines, is predicted by the untwisting hypothesis. The observed small offset suggests a possibility that in this event Cluster crosses the neutral sheet on the dawnside of the centre of the region of Earthward transported untwisting field lines (see discussion in Sect. 4).

\subsection{The flow event: SuperDARN}

In Fig. 8 we present two snapshots of the SuperDARN ionospheric convection observations, one for each hemisphere made at 11:28-11:30 UT i.e. during the fast flow event. The colour-coded vectors are the plasma flow vectors and the solid and dashed contours indicate the contours of the ionospheric electric potential, or equivalently, streamlines of plasma flow. The green line marks the low-latitude boundary of the ionospheric convection (zero-flow boundary or the Heppner-Maynard boundary below which the electric potential is assumed to be zero). Although the plasma convection in the ionospheric regions with no direct measurements are derived from a statistical model, the results can be considered as quite realistic due to the rather static IMF conditions within several hours before the event. Fortunately, the measured flow patterns exist in the most interesting regions of the ionosphere in both hemispheres for this event.

The ionospheric convection in both hemispheres show an asymmetric convection pattern. The morning convection cell is extending into the midnight sector in the Northern Hemisphere and the evening cell in the Southern Hemisphere. Note that the Southern Hemisphere convection pattern covers a smaller area than the northern pattern. This is plausibly owing to poor radar data coverage in the Southern Hemisphere. Since there must be same amount of open magnetic flux in the both polar caps, one might presume a similarly sized convection pattern in both hemispheres.

The red squares in Fig. 8 mark the ionospheric footprints of Cluster using T96 model. For clarity, only the C3 a)

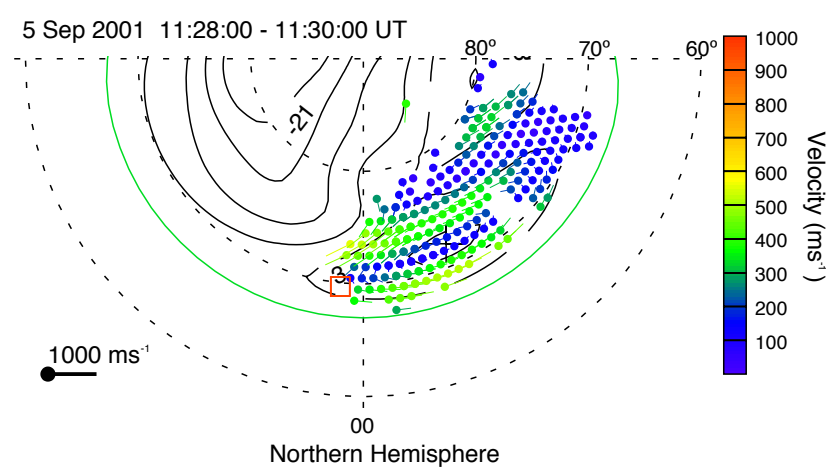

b)

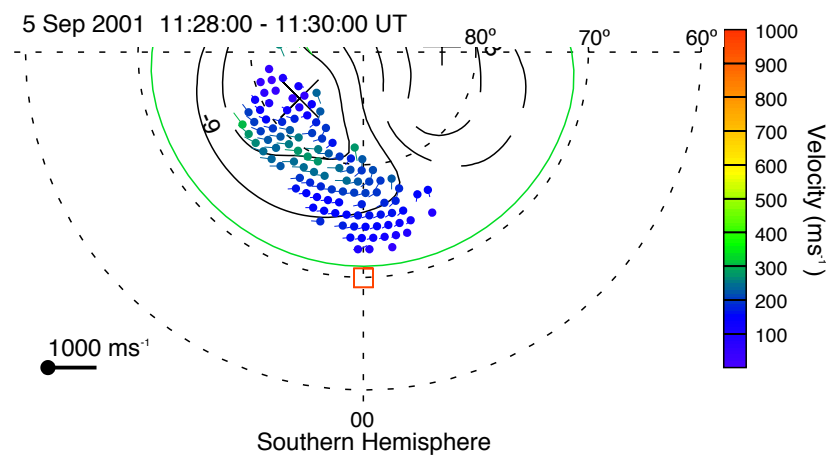

Figure 8. SuperDARN ionospheric plasma convection (a) in the Northern Hemisphere and (b) in the Southern Hemisphere in AACGM latitude - magnetic local time coordinate system on 5 September 2001 at 11:28-11:30 UT. The contours of the ionospheric electric potential are superposed by the black solid and dashed lines. The green line indicates the low-latitude boundary of the ionospheric convection. The T96 footprint of Cluster is marked by the red square.

footprints are shown (the footprints of other spacecraft are closely located). From Fig. 8a we note that the Cluster footprint in the Northern Hemisphere is mapped to the immediate vicinity of the enhanced eastward ionospheric plasma flows in the midnight sector. These eastward flows are consistent with the dawnward flow on northern field lines observed by Cluster in the magnetotail. In the Southern Hemisphere, the Cluster footprint is located slightly equatorward of the measured weak westward plasma flows (the flow is westward at all data points in the midnight sector even if it is somewhat difficult to distinguish it). It is reasonable to assume that the plasma flow is westward also at the Cluster footprint, since one might presume similarly sized convection patterns in both hemispheres, as discussed above, and the evening cell is dominating in the footprint region. This plasma flow could also be enhanced as the eastward flows in the Northern Hemisphere. In that case the enhanced westward flow would be consistent with the duskward flow measured by Cluster in southern field lines in the tail during the event. The iono- 
spheric convection patterns stayed nearly the same during the time interval the fast flow event was observed by Cluster.

However, it should be kept in mind that the Cluster footprints are from a model and represent the footprints of the same field line. If we assume that the ionospheric convection patterns in Fig. 8 are a manifestation of the tail untwisting process, one would expect the southern conjugate of the marked northern footprint to map to the morning convection cell well in the morning sector in the Southern Hemisphere (analogously as in Fig. 2). Similarly, the northern conjugate for the marked southern footprint would be expected to map to the evening cell in the evening sector in the Northern Hemisphere. Thus, it is clear that the actual tail twisting and the azimuthal displacement of the magnetic field line footprints must be much stronger and larger, respectively, than the T96 model suggests.

In conclusion, the SuperDARN measurements of ionospheric convection support the Cluster observations of the azimuthal flow shear across the neutral sheet. In addition, the measured flows in the magnetosphere and conjugate ionosphere are consistent with what one would expect if the flows were caused by the untwisting of twisted tail field lines. According to the untwisting hypothesis, for positive IMF $B_{y}$ conditions and hence for positive twisting of the tail field lines, the fast flows are expected to be predominantly eastward (dawnward) in the Northern Hemispheric and westward (duskward) in the Southern Hemispheric midnight ionosphere (magnetotail) (e.g. Grocott et al., 2005, 2007). That kind of situation is schematically illustrated in Figs. 1 and 2.

\section{Discussion}

The idea of tail untwisting has been suggested by Grocott et al. (2004) to explain the observations of fast azimuthal flow bursts seen in ionospheric convection in the midnight sector during predominantly northward and $B_{y}$ dominating IMF and nonsubstorm conditions. However, there has been a lack of magnetospheric observations that show the hypothesized untwisting process in action. Only the case study by Grocott et al. (2007) and to some extent also by Walsh et al. (2009) show magnetospheric measurements that could be related to or explained by the untwisting phenomenon. Grocott et al. (2007) report two fast flow events in which the convective azimuthal components match the conjugate ionospheric flows, which is in agreement with the untwisting hypothesis. In particular in their second event, Grocott et al. (2007) present observations of opposite azimuthal convection by two Cluster satellites from either side of the tail neutral sheet, which suggests an existence of an azimuthal flow shear across the neutral sheet. Walsh et al. (2009) report an azimuthal flow shear across the neutral sheet observed by only one of the four Cluster satellites in association with an Earthward fast flow, but they are not able to conclude whether the flow shear is related to tail untwisting or not, and do not discuss it any further.

The detailed multispacecraft magnetospheric observations presented in this paper confirm the previous indications of a possibility of an existence of an azimuthal flow shear across the neutral sheet within a fast flow and provide strong supportive evidence for the tail untwisting hypothesis: first, the Cluster observations show a positive tail $B_{y}$ component collinear with the concurrent IMF $B_{y}$ direction for several hours before the event. The ion data suggest that the collinearity occurs both on open and closed field lines, which proposes positive twisting of the tail magnetic field lines. In addition, the T96 model produces positively twisted closed field lines and the TF04 model an indication of a positively tilted neutral sheet for the event, which are in agreement with the Cluster observations. The actual plasma flow signatures within the fast flow show an azimuthal flow shear across the neutral sheet in the direction that would be expected in untwisting of positively twisted field lines. When comparing the flow shear with the conjugate ionospheric flows by SuperDARN we find that the directions of the azimuthal flow component in the opposite sides of the neutral sheet match to the flow directions of the ionospheric flows in the corresponding hemispheres. Furthermore, the ionospheric flow patterns resemble the flow patterns that have been previously attributed to the occurrence of untwisting (e.g. Grocott et al., 2005). The azimuthal directions within the fast flow are also consistent with the statistical results of Pitkänen et al. (2013) of IMF-dependent favourable azimuthal flow directions of convective fast flows, which they suggested possibly to be originated from untwisting.

The analysis of the flow shear reveals a small offset between the neutral sheet and the location at which the azimuthal flow reverses. This is the first time such an observation is reported. The flow reversal or the change of the $V_{\perp y}$ sign occurs slightly below the neutral sheet with an offset of $B_{x} \sim-0.4 \mathrm{nT}$. The untwisting hypothesis predicts an existence of such kind of offset. According to the hypothesis, the azimuthal flow reversal generated by the Earthward transported untwisting magnetic field lines is expected to be located at or close to the neutral sheet for untwisting field lines near local midnight. Farther away from midnight, i.e. towards the dawn and dusk flanks of the region of untwisting field lines, the flow reversal should be situated above or below the neutral sheet. Whether the reversal should be located below the neutral sheet in the dawnside and above in the duskside, or vice versa, depends on whether the tail is twisted positively or negatively, respectively. In the event studied in this paper, the flow reversal is located below the neutral sheet, which for positive tail twisting suggests a possibility that the neutral sheet crossing occurs in the dawnward part of the channel of the Earthward fast flow.

However, the $\sim-0.4 \mathrm{nT} B_{x}$ offset is small and its magnitude is comparable with (in fact smaller than) the standard deviation $\sim 0.5 \mathrm{nT}$ derived from the regression anal- 
ysis (red line in Fig. 7). Hence, one may argue that deducing the Cluster neutral sheet crossing region using the offset in the $B_{x}$ component would not be justified. On the other hand, there is a possibility that the plasma frame in the vicinity of the spacecraft could be moving dawnward at $V_{\perp y} \sim-24 \mathrm{~km} \mathrm{~s}^{-1}$, which would cause the offset.

The question also arises, whether the observed flow shear and azimuthally oppositely directed flows can be explained by any other mechanism. Walsh et al. (2009) suggested their event to be related to untwisting, but also discussed the possibility that the flows could be related to flow patterns internal to the fast flow channel (depleted bubble). They lacked SuperDARN ionospheric flow observations from both hemispheres and thus could not draw any definite conclusions regarding the cause for the shear in the flows. Grocott et al. (2007), on the other hand, had supportive ionospheric data available and concluded that the oppositely directed azimuthal flows in the magnetosphere in their event were due to untwisting. Fortunately, for our event, SuperDARN observations are available in both hemispheres, and they together with the Cluster observations give support to the untwisting scenario. The results of this study further suggest that the oppositely directed azimuthal flows above and below the neutral sheet are a global property of the channel of the Earthward fast flow and not any local disturbance within the flow pattern.

\section{Summary and conclusions}

In this study, we have presented detailed multispacecraft observations made by Cluster of an azimuthal velocity shear in the perpendicular flow component within an Earthward fast flow in the magnetotail. The fast flow event, which occurred on 5 September 2001, showed a clear flow shear velocity pattern, i.e. a $V_{\perp y}$ sign change, around the neutral sheet $\left(B_{x} \sim 0\right)$ associated with a fast flow during the neutral sheet flapping motion across the satellite locations. This suggests that the convection within the proper flow channel of a fast flow may not generally be azimuthally unidirectional across the neutral sheet, but may have a more complex structure.

The event was associated with northward and $B_{y}$ dominating IMF conditions without any signatures of substorm activity. However, the magnetospheric data measured by Cluster showed some signatures that could possibly be related to a twisting-associated stretching of the magnetotail before the event, analogously to a substorm growth phase. The observed fast flow would then be a natural manifestation of a localized tail relaxation process. Generally, both the Cluster observations and the T96 model suggest a twisting of closed field lines in the positive direction (positive $B_{y}$ collinear with the positive IMF $B_{y}$ ). In addition, the TF04 model indicates weak but consistent tilt of the neutral sheet.

During the fast flow, all the three Cluster satellites $(\mathrm{C} 1$, $\mathrm{C} 3$ and $\mathrm{C} 4)$ observed a change of the azimuthal velocity component from dawnward to duskward when moving from northern field lines to southern field lines as the neutral sheet moved in the +ZGSM direction over the satellites. The directions of the azimuthal flows at both sides of the neutral sheet are in agreement with that which would be expected for the flow directions if the flows were directed due to untwisting during the magnetic field reconfiguration following reconnection in a twisted tail (e.g. Grocott et al., 2005, 2007).

The flow reversal i.e. $V_{\perp y}$ sign change did not occur precisely at the neutral sheet but was displaced somewhat below it. At the reversal the $B_{x}$ component had a value of $\sim-0.4 \mathrm{nT}$. In addition, an indication of a linear dependence between the $B_{x}$ and $V_{\perp y}$ was found close to the neutral sheet $\left(-2 \leq B_{x} \leq 2 \mathrm{nT}\right)$. A linear fit gave a relation $V_{\perp y}=$ $-53.8 \cdot B_{x}-23.9$, where $V_{\perp y}$ and $B_{x}$ are given in $\mathrm{km} \mathrm{s}^{-1}$ and $\mathrm{nT}$, respectively.

This observation of an offset between the neutral sheet and the flow reversal is the first reported in the literature. However, the untwisting hypothesis predicts an existence of such kind of offset. Towards the dawn and dusk flanks of the flow channel the flow reversal is expected to be situated above or below the neutral sheet depending on the tail twisting direction. The observations provide us with the opportunity to infer the neutral sheet crossing region in respect to the dawndusk extent of the fast flow channel in the framework of the untwisting hypothesis. In this event, the neutral sheet crossing was inferred to occur in the dawnward part of the channel of the Earthward fast flow. However, we cannot rule out uncertainties associated with the offset (smaller than the standard deviation) nor other explanations like the possibility of motion of the plasma frame in the vicinity of the spacecraft as factors that could affect the correctness of our inference. The linear dependence between the $B_{x}$ and $V_{\perp y}$ close to the neutral sheet within the fast flow is also an interesting new finding and it should be investigated whether this relation is a typical feature for fast flows during nonzero IMF $B_{y}$ conditions.

During the event, ionospheric convection observed by the SuperDARN radars showed enhanced eastward plasma flows in the Northern Hemisphere and plausible westward flows in the Southern Hemisphere in the conjugate regions. This is consistent with the azimuthal flow directions measured by Cluster. However, obviously, there exists a problem with proper mapping of the Cluster measurements into the ionosphere. In this event, the T96 mapping provided a clearly too small azimuthal displacement for mapped footprints due to T96 model underestimation of the true twisting in the magnetotail. However, the ionospheric observations resemble those flow patterns previously attributed to the untwisting of twisted tail field lines (e.g. Grocott et al., 2005, 2007) supporting the possibility that the flows measured by Cluster were due to untwisting. Hence, by combining all evidence provided by the data presented here, we suggest that the fast flows observed in the magnetosphere were a manifestation of the untwisting of twisted tail field lines, although the proper 
ionospheric mapping of magnetospheric observations from a twisted tail remains a problem. Thus, fully consistent conclusions cannot be drawn.

In conclusion, the event presented in this paper provides strong supportive evidence for the untwisting hypothesis which explains the occurrence of azimuthally directed flows in the nightside ionosphere and magnetosphere by untwisting of twisted magnetic field lines during nonzero IMF $B_{y}$ conditions. Albeit our observations show a clear multispacecraft example of an azimuthal flow shear across the neutral sheet within a fast flow, more observations are needed to establish whether the flow shears with reversals are an essential feature of the structure of the fast flows.

Acknowledgements. The authors thank the CIS and FGM teams and the Cluster Active Archive for the Cluster data and GSFC SPDF/OMNIWeb for the solar wind data. The authors also acknowledge the PIs of the SuperDARN radars for the radar data. WDC for Geomagnetism, Kyoto, is acknowledged for the AE index data. The work by T. Pitkänen and M. Hamrin was supported by the Swedish National Space Board project 78/11AB.

Topical Editor C.-P. Escoubet thanks V. A. Sergeev and one anonymous referee for their help in evaluating this paper.

\section{References}

Balogh, A., Carr, C. M., Acuña, M. H., Dunlop, M. W., Beek, T. J., Brown, P., Fornacon, K.-H., Georgescu, E., Glassmeier, K.H., Harris, J., Musmann, G., Oddy, T., and Schwingenschuh, K.: The Cluster Magnetic Field Investigation: overview of in-flight performance and initial results, Ann. Geophys., 19, 1207-1217, doi:10.5194/angeo-19-1207-2001, 2001.

Chisham, G., Lester, M., Milan, S. E., Freeman, M. P., Bristow, W. A., Grocott, A., McWilliams, K. A., Ruohoniemi, J. M., Yeoman, T. K., Dyson, P. L., Greenwald, R. A., Kikuchi, T., Pinnock, M., Rash, J. P. S., Sato, N., Sofko, G. J., Villain, J.P., and Walker, A. D. M.: A decade of the Super Dual Auroral Radar Network (SuperDARN): Scientific achievements, new techniques and future directions, Surv. Geophys., 28, 33-109, doi:10.1007/s10712-007-9017-8, 2007.

Cowley, S. W. H.: Magnetospheric asymmetries associated with the Y-component of the IMF, Planet. Space Sci., 29, 79-96, doi:10.1016/0032-0633(81)90141-0, 1981.

Fairfield, D. H.: On the average configuration of the geomagnetic field, J. Geophys. Res., 84, 1950-1958, doi:10.1029/JA084iA05p01950, 1979.

Greenwald, R. A., Baker, K. B., Dudeney, J. R., Pinnock, M., Jones, T. B., Thomas, E. C., Villain, J.-P., Cerisier, J.-C., Senior, C., Hanuise, C., Hunsucker, R. D., Sofko, G., Koehler, J., Nielsen, E., Pellinen, R., Walker, A. D. M., Sato, N., and Yamagishi, H.: Darn/SuperDarn: A global view of the dynamics of high-latitude convection, Space Sci. Rev., 71, 761-796, doi:10.1007/BF00751350, 1995.

Grocott, A., Cowley, S. W. H., and Sigwarth, J. B.: Ionospheric flow during extended intervals of northward but $B_{y}$-dominated IMF, Ann. Geophys., 21, 509-538, doi:10.5194/angeo-21-509-2003, 2003.
Grocott, A., Badman, S. V., Cowley, S. W. H., Yeoman, T. K., and Cripps, P. J.: The influence of IMF By on the nature of the nightside high-latitude ionospheric flow during intervals of positive IMF $B_{z}$, Ann. Geophys., 22, 1755-1764, doi:10.5194/angeo-221755-2004, 2004.

Grocott, A., Yeoman, T. K., Milan, S. E., and Cowley, S. W. H.: Interhemispheric observations of the ionospheric signature of tail reconnection during IMF-northward non-substorm intervals, Ann. Geophys., 23, 1763-1770, doi:10.5194/angeo-231763-2005, 2005.

Grocott, A., Yeoman, T. K., Milan, S. E., Amm, O., Frey, H. U., Juusola, L., Nakamura, R., Owen, C. J., Rème, H., and Takada, T.: Multi-scale observations of magnetotail flux transport during IMF-northward non-substorm intervals, Ann. Geophys., 25, 1709-1720, doi:10.5194/angeo-25-1709-2007, 2007.

Grocott, A., Milan, S. E., and Yeoman, T. K.: Interplanetary magnetic field control of fast azimuthal flows in the nightside high-latitude ionosphere, Geophys. Res. Lett., 35, L08102, doi:10.1029/2008GL033545, 2008.

Kaymaz, Z., Siscoe, G. L., Luhmann, J. G., Lepping, R. P., and Russell, C. T.: Interplanetary magnetic field control of magnetotail field geometry: IMP 8 observations, J. Geophys. Res., 99, 11113-11126, doi:10.1029/94JA00300, 1994.

Kaymaz, Z., Siscoe, G., Luhmann, J. G., Fedder, J. A., and Lyon, J. G.: Interplanetary magnetic field control of magnetotail field: IMP 8 data and MHD model compared, J. Geophys. Res., 100, 17163-17172, doi:10.1029/95JA00593, 1995.

Kullen, A. and Janhunen, P.: Relation of polar auroral arcs to magnetotail twisting and IMF rotation: a systematic MHD simulation study, Ann. Geophys., 22, 951-970, doi:10.5194/angeo-22-9512004, 2004a.

Kullen, A. and Janhunen, P.: Erratum "Relation of polar auroral arcs to magnetotail twisting and IMF, rotation: a systematic MHD simulation study", Ann. Geophys., Ann. Geophys., 22, 26552655, doi:10.5194/angeo-22-2655-2004, 2004b.

Lui, A. T. Y.: Polar wind influence on magnetotail configuration and dynamics, in: Solar wind magnetosphere coupling, edited by: Kamide, Y. and Slavin, J. A., 671, 1986.

Owen, C. J., Slavin, J. A., Richardson, I. G., Murphy, N. and Hynds, R. J.: Average motion, structure and orientation of the distant magnetotail determined from remote sensing of the edge of the plasma sheet boundary layer with $E>35 \mathrm{keV}$ ions, J. Geophys. Res., 100, 185-204, doi:10.1029/94JA02417, 1995.

Petrukovich, A. A., Baumjohann, W., Nakamura, R., Schödel, R., and Mukai, T.: Are earthward bursty bulk flows convective or field-aligned, J. Geophys. Res., 106, 21221-21215, doi:10.1029/2001JA900019, 2001.

Pitkänen, T., Hamrin, M., Norqvist, P., Karlsson, T., and Nilsson, H.: IMF dependence of the azimuthal direction of earthward magnetotail fast flows, Geophys. Res. Lett., 40, 5598-5604, doi:10.1002/2013GL058136, 2013.

Raj, A., Phan, T., Lin, R. P., and Angelopoulos, V.: Wind survey of high-speed bulk flows and field-aligned beams in the near-Earth plasma sheet, J. Geophys. Res., 107, 1419, doi:10.1029/2001JA007547, 2002.

Rème, H., Aoustin, C., Bosqued, J. M., Dandouras, I., Lavraud, B., Sauvaud, J. A., Barthe, A., Bouyssou, J., Camus, Th., Coeur-Joly, O., Cros, A., Cuvilo, J., Ducay, F., Garbarowitz, Y., Medale, J. L., Penou, E., Perrier, H., Romefort, D., Rouzaud, J., Vallat, C., 
Alcaydé, D., Jacquey, C., Mazelle, C., d'Uston, C., Möbius, E., Kistler, L. M., Crocker, K., Granoff, M., Mouikis, C., Popecki, M., Vosbury, M., Klecker, B., Hovestadt, D., Kucharek, H., Kuenneth, E., Paschmann, G., Scholer, M., Sckopke, N., Seidenschwang, E., Carlson, C. W., Curtis, D. W., Ingraham, C., Lin, R. P., McFadden, J. P., Parks, G. K., Phan, T., Formisano, V., Amata, E., Bavassano-Cattaneo, M. B., Baldetti, P., Bruno, R., Chionchio, G., Di Lellis, A., Marcucci, M. F., Pallocchia, G., Korth, A., Daly, P. W., Graeve, B., Rosenbauer, H., Vasyliunas, V., McCarthy, M., Wilber, M., Eliasson, L., Lundin, R., Olsen, S., Shelley, E. G., Fuselier, S., Ghielmetti, A. G., Lennartsson, W., Escoubet, C. P., Balsiger, H., Friedel, R., Cao, J.-B., Kovrazhkin, R. A., Papamastorakis, I., Pellat, R., Scudder, J., and Sonnerup, B.: First multispacecraft ion measurements in and near the Earth's magnetosphere with the identical Cluster ion spectrometry (CIS) experiment, Ann. Geophys., 19, 1303-1354, doi:10.5194/angeo19-1303-2001, 2001.

Ruohoniemi, J. M. and Baker, K. B.: Large-scale imaging of highlatitude convection with Super Dual Auroral Radar Network HF radar observations, J. Geophys. Res., 103, 20797-20811, doi:10.1029/98JA01288, 1998.

Ruohoniemi, J. M. and Greenwald, R. A.: Statistical patterns of high-latitude convection obtained from Goose Bay HF radar observations, J. Geophys. Res., 101, 21743-21764, doi:10.1029/96JA01584, 1996.

Russell, C. T.: The configuration of the magnetosphere, in: Critical Problems of Magnetospheric Physics, edited by: Dyer, E. R., National Academy of Sciences, Washington, DC, 1, 1972.

Sergeev, V., Runov, A., Baumjohann, W., Nakamura, R., Zhang, T. L., Balogh, A., Louarnd, P., Sauvaud, J.-A., and Reme, H.: Orientation and propagation of current sheet oscillations, Geophys. Res. Lett., 31, L05807, doi:10.1029/2003GL019346, 2004.
Tsyganenko, N. A.: Modeling the Earth's magnetospheric magnetic field confined within a realistic magnetopause, J. Geophys. Res., 100, 5599-5612, doi:10.1029/94JA03193, 1995.

Tsyganenko, N. A. and Fairfield, D. H.: Global shape of the magnetotail current sheet as derived from Geotail and Polar data, J. Geophys. Res., 109, A03218, doi:10.1029/2003JA010062, 2004.

Tsyganenko, N. A., Karlsson, S. B. P., Kokubun, S., Yamamoto, T., Lazarus, A. J., Ogilvie, K. W., and Russell, C. T.: Global configuration of the magnetotail current sheet as derived from Geotail, Wind, IMP 8 and ISEE 1/2 data, J. Geophys. Res., 103, 68276841, doi:10.1029/97JA03621, 1998.

Walker, R. J., Richard, R. L., Ogino, T., and Ashour-Abdalla, M.: The response of the magnetotail to changes in the IMF orientation: The magnetotail's long memory, Phys. Chem. Earth C, 24, 221-227, 1999.

Walsh, A. P., Fazakerley, A. N., Lahiff, A. D., Volwerk, M., Grocott, A., Dunlop, M. W., Lui, A. T. Y., Kistler, L. M., Lester M., Mouikis, C., Pu, Z., Shen, C., Shi, J., Taylor, M. G. G. T., Lucek, E., Zhang, T. L., and Dandouras, I.: Cluster and Double Star multipoint observations of a plasma bubble, Ann. Geophys., 27, 725-743, doi:10.5194/angeo-27-725-2009, 2009.

Zhang, T. L., Baumjohann, W., Nakamura, R., Balogh, A., and Glassmeier, K.-H.: A wavy twisted neutral sheet observed by CLUSTER, Geophys. Res. Lett., 29, 1899-1902, doi:10.1029/2002GL015544, 2002.

Zhang, T. L., Nakamura, R., Volwerk, M., Runov, A., Baumjohann, W., Eichelberger, H. U., Carr, C., Balogh, A., Sergeev, V., Shi, J. K., and Fornacon, K.-H.: Double Star/Cluster observation of neutral sheet oscillations on 5 August 2004, Ann. Geophys., 23, 2909-2914, doi:10.5194/angeo-23-2909-2005, 2005. 